\title{
Futebol brasileiro e ciência: o campeonato da série A realmente é melhor do que a série $B$ ?
}

Brazilian soccer and science: is the A series really better than the B series?

\author{
Paulo Sérgio Machado Rodrigues ${ }^{1, *}$ \\ Gustavo Ribeiro da Mota ${ }^{1}$
}

\section{Resumo:}

Objetivo: Este estudo comparou variáveis quantitativas (passes, finalizações, desarmes, cruzamentos, lançamentos, dribles, gols, faltas, cartões, impedimentos e pênaltis) de jogos das duas divisões do Campeonato Brasileiro no ano de 2013. Métodos: Os dados foram coletados a partir de dois distintos portais eletrônicos especializado em estatística para futebol (www.footstats.net e www.globoesporte.com). Foram analisados 380 jogos em cada divisão, totalizando 760 partidas. As variáveis foram comparadas por meio de teste $T$ independente, com significância de $5 \%$. Também analisamos o tamanho do efeito (TE) de Cohen d. Resultados: As seguintes variáveis diferiram entre as séries $A$ e $B(p<0,05)$ : passes certos, passes errados, passes totais, percentual de sucesso em passes, percentual de sucesso em cruzamentos, percentual de sucesso em lançamentos e dribles errados (TE moderado para todas as variáveis). Houve superioridade $(p<0,05)$ da série $B$ em relação à série A para as variáveis finalizações certas, finalizações erradas, finalizações totais, percentual de sucesso de desarmes, percentual de sucesso de dribles, cartões amarelos, cartões vermelhos e faltas cometidas, (TE variando de pequeno a grande). Conclusão: Podemos concluir que a série $A$ apresenta melhor índice técnico, maior dificuldade em driblar e finalizar corretamente em relação à série $B$. Adicionalmente, a série $B$ é mais violenta do que a série $A$.

Palavras-chave: futebol, campeonato brasileiro, scout, análise estatística, variáveis qualitativas.

\begin{abstract}
:
Objective: The present study aimed to compare the quantitative variables (pass, completions, tackles, crosses, releases, dribbles, goals, fouls, cards, deterrents and penalties) of matches from two divisions of the Brazilian Championship in 2013. Methods: Data were collected from two distinct electronic portals specializing in statistics for soccer (www.footstats.net and www.globoesporte.com). It was analyzed 380 matches from each division, with a total of 760 matches. Variables were compared using an independent $t$ test with significance level of $5 \%$ also analyzed effect size (ES). Results: The results showed higher ( $p$ $<0.05$ ) difference in favor to division $A$ in relation division $B$ for the variables passes right, wrong passes and total passes, percentage of successful pass, percentage of successful crosses, percentage of successful long passes, and wrong dribbling's, with moderate ES for all variables. There were a significant superiority $(\mathrm{p}<0.05)$ for $\mathrm{B}$ division in relation to $\mathrm{A}$ division for correct, wrong and total shots success percentage of tackles and dribbling, yellow and red cards, and faults (ES - small to large). Conclusion: We can conclude that A division of Brazilian soccer shows better technical index, and is more difficult to dribble and to shot properly than $B$ division. Besides, $B$ division is more violent than $A$ division.
\end{abstract}

Keywords: soccer, brazilian championship, scout, statistical analysis, qualitative variables.

\section{Afiliação dos autores}

Universidade Federal do Triângulo Mineiro, Minas Gerais, Brasil

${ }^{*}$ Autor correspondente

Rua Frei Paulino, № 30 - Bairro Abadia, Uberaba/, Minas Gerais, Brasil, Cep:38025-180

E-mail:ps_mrodrigues@hotmail.com

Conflito de interesses

Os autores declararam não haver conflito de interesses.

Processo de arbitragem

Recebido: 19/03/2018 Aprovado: 13/08/2018 


\section{Introdução}

Os jogos desportivos coletivos (JDC) de invasão ao campo adversário se caracterizam por ocorrer dentro de contexto aleatório e dinâmico, com oposição aos adversários e cooperação aos membros da mesma equipe, tais como futsal, futebol handebol, basquetebol e rugby ${ }^{1}$.Dentro deste contexto, o futebol oferece vasto campo de estudos, sejam eles físicos/fisiológicos $^{2-5}$, preventivos $^{6,7}$, motivacionais ${ }^{8}$ e sociais $^{9}$, em diferentes subtipos de futebol ${ }^{10-13}$, ou até mesmo quantificando dados técnicos para melhor compreensão científica ${ }^{5,14}$ e eventual aplicação prática.

O futebol apresenta diversas variáveis técnico/táticas que podem ser analisadas no decorrer de uma partida, tais como passes, finalizações a gol, cruzamentos e gols, forma de jogar da equipe, padrão de jogo, que seria basicamente aspectos táticos que se repetem em todas as partidas da equipe como adoção de esquemas do tipo $4: 4: 2$ ou 3:5:2, bem como a atitude permanente dos jogadores em relação aos aspectos táticos ${ }^{1}$.

Pensando nesta aleatoriedade de contextos, o futebol pode ser quantificado e até mesmo qualificado por uma ferramenta que vem ganhando notoriedade atualmente: o Scout ${ }^{15}$. O Scout ou análise estatística pode ser descrito como o mapeamento de ações que ocorrem em uma partida, seja ele individualizado ou em equipe ${ }^{16}$. A partir de tais análises, podemos ter outra visão sobre o que ocorre em determinada partida, ou até mesmo o que realmente aconteceu ao longo de um campeonato inteiro. Tais dados são importantes para as equipes, gestores das competições, imprensa e, talvez, até mesmo para os espectadores.

Mesmo com o avanço tecnológico e o surgimento de grande quantidade de dados de jogos (ex. chutes, posse de bola, passes certos) disponíveis pela TV, por exemplo, surpreendentemente pouco se conhece sobre as reais diferenças (quantitativas) entre campeonatos de diferentes níveis. Ademais, estudos em ciências do esporte precisam atender ao princípio da especificidade da modalidade esportiva $^{17-19}$. Ou seja, não há algo mais específico que o estudo do próprio jogo. Apesar disso, o senso comum, algo até compreensível pela lógica, de torcedores, admiradores e imprensa em geral é que, por exemplo, existem diferenças significativas entre os Campeonatos Brasileiros da Série A e da Série B. Porém, desconhecemos estudos que tenham analisado eventuais diferenças de maneira científica. Assim, o presente estudo teve por objetivo analisar e comparar indicadores técnicos quantitativos das duas principais divisões do futebol brasileiro (dados de 2013). Nossa hipótese foi que, de alguma maneira, a divisão de elite (séria A) apresentasse melhores índices técnicos do que a série $B$.

\section{Métodos}

\section{Amostra}

A amostra foi composta por $100 \%$ das partidas realizadas durante os Campeonatos Brasileiros da Série A e B do ano de 2013, totalizando 760 jogos (380 jogos de cada série) das 40 equipes participantes das duas competições (20 equipes da série $A$ e 20 equipes da série $B$ ), resultando em 76 rodadas provenientes das duas divisões (38 de cada divisão).

\section{Indicadores Técnicos}

Os dados foram obtidos por meio de dois portais eletrônicos distintos, Footstats.com e Globoesporte.com. Todos os dados foram alojados em planilhas do Microsoft Excel 2010 para posterior análise.

\section{Análise Estatística}

Testamos a distribuição dos dados por meio do teste D'Agostino e Pearson. Quando as variáveis apresentaram distribuição normal, utilizamos o teste $t$-studentpara amostras independentes (análise paramétrica). Quando a distribuição foi considerada não normal, aplicamos o teste não paramétrico de Mann Whitney. Quando apropriado, testamos as correlações entre as variáveis (Pearson para dados paramétricos e Spearman para não paramétricos) $O$ tamanho do efeito (TE) foi calculado para determinar a magnitude da diferença ${ }^{20}$ sobre as variáveis. A magnitude do TE foi classificada como trivial $(<0,2)$, pequena $(>0,2-0,6)$, moderada $(>0,6-1,2)$, grande $(>1,2-2,0)$ e muito grande $(>2,0-4,0)$ baseado nas diretrizes de Batterham e Hopkins $^{21}$. O nível de significância adotado foi de $p<0,05$. O programa estatístico utilizado para análise dos dados e confecção das figuras foi GraphPad ( Prism, 6.0, San Diego, USA.).

\section{Resultados}

Um resumo de todas as variáveis analisadas, incluindo os valores de " $p$ " e o TE, comparadas entre as séries A e B encontram-se na Tabela 1. As variáveis relacionadas aos passes (certos, errados, totais e percentual de sucesso [\%sucesso]) foram todas superiores $(p<0,05, \mathrm{TE}=$ moderado) para a série $A$ em relação à série $B$. Em relação às finalizações, a série $B$ apresentou maiores valores $(p<0,05, T E=$ pequeno a moderado) do que a série $A$, exceto para o \% de sucesso nesse fundamento que não diferiu ( $p>0,05, T E=$ trivial).Por sua vez, $o$ fundamento desarme não diferiu ( $p>0,05$, TE = pequeno) em termos absolutos (certos, errados e totais) entre as séries, mas o $\%$ de sucesso foi maior ( $p<0,05$, TE = moderado) para a série $B$ do que para a série A.No tocante aos cruzamentos, os valores absolutos (certos, errados e totais) não diferiram $(p>0,05, \mathrm{TE}=$ trivial a pequeno) entre as séries mas o aproveitamento (\% de sucesso) foi maior ( $p<0,05, T E=$ moderado) para a série $A$ do que a série $B$. Igualmente, os lançamentos apresentaram o mesmo padrão dos cruzamentos. Ou seja, valores absolutos semelhantes $(p>0,05, T E=$ trivial a pequeno) entre as séries, mas o \% sucesso foi superior $(p<0,05$, TE $=$ moderado) para $a$ séria $A$ em relação à séria $B$. No que concerne aos dribles, não houve diferença $(p>0,05, T E=$ trivial a pequeno) entre as séries para os dribles certos e totais. Porém, interessantemente, a série $A$ apresentou maior $(p<0,05, T E=$ moderado) quantidade de dribles errados do que a série $B$ e a série $B$, por outro lado, aproveitou melhor as oportunidades de dribles do que a série $A$ (\% sucesso maior, $p<0,05$, TE = grande).

O número de gols marcados não foi diferente $(p>0,05, T E=$ pequeno) entre as séries. Entretanto, o número de cartões amarelos, vermelhos e de faltas foi mais elevado $(p<0,05, T E=$ moderado) para a série $B$ do que para a série $A$. Inclusive encontramos fortes correlações ( $r$ entre 0,96 e 0,99 e $p<0,05$ ) entre quantidade de cartões e número de faltas para ambas as séries (Tabela 2).Não houve diferenças ( $p>0,05$, TE trivial a pequeno) entre o número de impedimentos bem como de pênaltis cometidos entre as séries $A$ e $B$.

Tabela 1

Resumo da análise estatística realizada comparando as variáveis de jogo analisadas no presente estudo.

\begin{tabular}{|c|c|c|c|}
\hline Variável & $P$ & TE & Classificação TE \\
\hline Passes Certos ${ }^{*}$ & 0,0059 & 0,92 & Moderado \\
\hline Passes Errados* & 0,0040 & 0,97 & Moderado \\
\hline Passes Totais ${ }^{*}$ & 0,0056 & 0,93 & Moderado \\
\hline Percentual de Sucesso* & 0,0146 & 0,84 & Moderado \\
\hline Finalizações Certas* & 0,0164 & 0,58 & Pequeno \\
\hline Finalizações Erradas* & 0,0050 & 0,95 & Moderado \\
\hline Finalizações Totais* ${ }^{*}$ & 0,0149 & 0,81 & Moderado \\
\hline Percentual de Sucesso & 0,3933 & 0,19 & Trivial \\
\hline Desarmes Certos & 0,3969 & 0,27 & Pequeno \\
\hline Desarmes Errados & 0,0725 & 0,58 & Pequeno \\
\hline Desarmes Totais & 0,2866 & 0,34 & Pequeno \\
\hline Percentual de Sucesso* & 0,0012 & 1,12 & Moderado \\
\hline Cruzamentos Certos & 0,6638 & 0,14 & Trivial \\
\hline Cruzamentos Errados & 0,1554 & 0,46 & Pequeno \\
\hline Cruzamentos Totais & 0,3529 & 0,30 & Pequeno \\
\hline Percentual de Sucesso* & 0,0005 & 0,90 & Moderado \\
\hline Lançamentos Certos & 0,1539 & 0,46 & Pequeno \\
\hline Lançamentos Errados & 0,6194 & 0,16 & Trivial \\
\hline Lançamentos Totais & 0,3709 & 0,29 & Pequeno \\
\hline Percentual de Sucesso* & 0,0288 & 0,72 & Moderado \\
\hline Dribles Certos & 0,1894 & 0,05 & Trivial \\
\hline Dribles Errados* & 0,0210 & 0,76 & Moderado \\
\hline Dribles Totais & 0,1291 & 0,26 & Pequeno \\
\hline Percentual de Sucesso* & $<0,0001$ & 1,67 & Grande \\
\hline Gols Marcados & 0,4162 & 0,26 & Pequeno \\
\hline Cartões Amarelos* & 0,0007 & 1,17 & Moderado \\
\hline Cartões Vermelhos* & 0,0257 & 0,74 & Moderado \\
\hline Faltas Cometidas* & 0,0084 & 0,88 & Moderado \\
\hline Impedimentos & 0,2302 & 0,39 & Pequeno \\
\hline Pênaltis & 0,5734 & 0,19 & Trivial \\
\hline
\end{tabular}

Tabela 2 
Correlações $(r)$ fortes $(p<0,01)$ foram encontradas entre os números de cartões amarelos e vermelhos com a quantidade de faltas cometidas para ambas as séries ( $A$ e $B)$.

\begin{tabular}{cccc}
\hline & $\begin{array}{c}\text { Cartões } \\
\text { Amarelos }\end{array}$ & $\begin{array}{c}\text { Cartões } \\
\text { Vermelhos }\end{array}$ & $\begin{array}{c}\text { Faltas } \\
\text { Cometidas }\end{array}$ \\
\hline $\begin{array}{c}\text { Cartões } \\
\text { Amarelos A } \\
\text { Cartões }\end{array}$ & - & 0,96 & 0,96 \\
$\begin{array}{c}\text { Vermelhos A } \\
\text { Faltas }\end{array}$ & 0,96 & - & 0,98 \\
$\begin{array}{c}\text { Cometidas A } \\
\text { Cartões }\end{array}$ & 0,96 & 0,98 & - \\
$\begin{array}{c}\text { Amarelos B } \\
\text { Cartões } \\
\text { Vermelhos B } \\
\text { Faltas }\end{array}$ & - & 0,97 & 0,99 \\
Cometidas B & 0,97 & - & 0,97 \\
\hline
\end{tabular}

\section{Discussão}

O presente estudo teve como objetivo principal evidenciar possíveis diferenças entre a série $A$ e a série $B$ do Campeonato Brasileiro. Nossos principais achados foram que a série $A$ apresenta melhor índice técnico e a série B exibe jogos com maior número de interrupções e com inferior índice técnico em relação à série $A$. Tal fato corrobora nossa hipótese inicial.

Evidenciar as diferenças entre competições pode ser uma forma coerente de responder a várias questões, que vão desde suas exigências, passando por informações importantes para as equipes técnicas e de gestores das mesmas. Até o torcedor que discute trivialmente os resultados de um jogo ou a diferença de um campeonato para o outro.

Em relação aos passes, a série $A$ apresentou maior número de passes totais em relação à série $B$. Supomos que quanto mais vezes um evento acontece, maior a chance de este evento ser correto ou não, o que nos leva a entender o também maior número de passes certos e errados da série $A$ em relação à série $B$. Em relação à eficiência, entretanto, a porcentagem de sucesso de passes da série $A$ se mostrou superior à da série $B$, o que nos remete acreditar que a série $A$ apresenta jogo mais técnico, com maior volume. Ou seja, maior quantidade de passes certos associada ao maior aproveitamento dos mesmos (\%sucesso) refletem maior eficiência e melhor qualidade dos jogadores.

Comparados com os estudos realizados ${ }^{16}$, em que algumas variáveis da equipe de futebol da Associação Atlética Ponte Preta foram analisadas por meio de duas diferentes competições, campeonato paulista e campeonato brasileiro, dos anos de 1997 até o ano de 1999, onde observaram certa regularidade em relação aos passes certos e errados da equipe nas duas competições, no nosso estudo o mesmo não se confirmou em relação às variáveis de passe. Considerando o TE, obtivemos classificação de moderado nas quatro variáveis em relação aos passes. Isso pode ser entendido, na prática, que além de ser diferente em relação à série $\mathrm{A}$ e $\mathrm{B}$, essa diferença é moderadamente importante. Infelizmente, não encontramos nenhum estudo comparativo, de diferentes níveis de campeonatos internacionais, para confrontarmos em relação ao atual trabalho.

Em relação às finalizações, a série $B$ apresentou valores absolutos superiores aos da série $A$, mas o relativo (\% de sucesso) não diferiu. Novamente, a quantidade superior de eventos ocorridos pode explicar tais fatos. Porém, especulamos que outra causa seria uma suposta ineficiência por parte dos jogadores nos sistemas defensivos das equipes da série $B$. Isso levaria a maior facilidade de finalização por parte dos atacantes, acarretando, portanto, superior número de finalizações. Outra possível explicação, embora não possamos afirmar com bases nos dados apresentados, é que na série $B$ pode ocorrer maior predominância do jogo direto em detrimento ao jogo indireto. O jogo direto (vertical, com objetivo de rapidamente finalizar) ${ }^{1}$, desencadeia maior número de tentativas "verticais" para marcar gols do que a série $A$, onde a melhor capacidade técnica/tática dos jogadores dificulta tal ação. Para 0 TE, a variável finalizações certas recebeu classificação de pequena. As variáveis finalizações erradas e finalizações totais receberam uma classificação de moderada, e a variável percentual de sucesso nas finalizações recebeu a classificação de trivial, o que nos leva a entender que na prática, há uma diferença real de finalizações certas e erradas da série $B$ em relação à série $A$, porém se formos observar o percentual de sucesso tal fator não se aplica à mesma.

Para Vendite, Vendite e Moraes $^{16}$, as finalizações podem determinar o resultado de uma partida, e corroborando com os achados do presente estudo, os valores encontrados pelos autores em duas diferentes competições foram similares.

Os gols marcados, não apresentaram diferenças significativas em comparação às duas séries, o que vem ao encontro de alguns estudos ${ }^{15}$, em que foram analisadas as médias de gols marcados em quatro diferentes campeonatos: Premier League (Inglaterra), Bundesliga (Alemanha), Série A (Itália) e La Liga (Espanha). Apesar de no presente estudo compararmos diferentes divisões de uma mesma competição, os dados do estudo citado demonstram que basicamente um mesmo número de gols é marcado em todas as quatro competições estudadas, o que ainda, segundo os autores, pode ser aplicado para os mais diversos campeonatos de alto nível em todo o mundo.

Com número maior de faltas cometidas na série $B$ do que na séria $A$, obviamente esperaríamos maior número de cartões (na série $B$ ), fato que também ocorreu com magnitude moderada. Isso significa que, realmente a série B apresenta jogos mais faltosos. Isto pode ser entendido como mais violento e com maior número de interrupções. Inclusive, encontramos forte correlação entre número de faltas e quantidade de cartões (amarelos e vermelhos), tanto na séria A quanto na série $B$. Esse achado deixa claro que faltas e cartões estão associados independentemente do nível (isto é, série A ou B). Assim, como na $B$ houve mais faltas, houve também superioridade em termos de cartões amarelos e vermelhos o que torna o jogo da série $B$ menos atrativo do ponto de vista técnico. Tais dados (de maior violência em séries inferiores) são similares aos encontrados ${ }^{15}$ em divisões de acesso do Campeonato Inglês. Ou seja, esses autores relataram maior número de cartões amarelos e vermelhos e maior número de faltas por jogo nas séries inferiores.

Algumas limitações do presente estudo precisam ser apresentadas para o leitor: o futebol é altamente complexo envolvendo vários outros aspectos como, por exemplo, físicos, técnicos/táticos, emocionais, ambientais, motivacionais, dentre outros fatores. Ademais, há tendência mundial em se analisar o esporte (não apenas o futebol) com abordagens mais holísticas, qualitativas $^{22}$. Portanto, obviamente, a análise quantitativa é limitada. Adicionalmente a exatidão dos dados reportados pelos dois provedores dos dados analisados não foi testada cientificamente o que, certamente, exige cautela com a interpretação aqui apresentada. Apesar dessas limitações, acreditamos que este estudo é "um primeiro passo" em direção às discussões mais robustas metodologicamente sobre 0 complexo cenário do desempenho no futebol.

\section{Conclusão}

Considerando os dados aqui apresentados, concluímos que a série $A$ do campeonato brasileiro de futebol apresenta jogos com melhores índices técnicos e menos truncados do que os jogos da série B. Adicionalmente, a indisciplina também é superior nos jogos da série $B$ em comparação com a série $A$. Apesar da aparente obviedade destas conclusões, este é o primeiro estudo a analisar essas informações sob a luz da ciência e permite futuras direções para pesquisas mais robustas sobre o complexo fenômeno denominado futebol.

\section{Referências}

1. Garganta J. Competências no ensino e treino de jovens futebolistas Lecturas: Educación Física y Deportes [Internet]. 2002; 8(45):[1-3 pp.].

2. Bradley PS, Di Mascio M, Peart D, Olsen P, Sheldon B. High-intensity activity profiles of elite soccer players at different performance levels. $J$ Strength Cond Res. 2010;24(9):2343-51.

3. Stølen T, Chamari K, Castagna C, Wisløff U. Physiology of soccer: an update. Sports Med. 2005;35(6):501-36.

4. Lopes CR, Crisp AH, Germano MD, de Mattos RS, Sindorf MAG, da Mota $\mathrm{GR}$, et al. Effects of pre-season short-term daily undulating periodized training on muscle strength and sprint performance of under-20 soccer players. International Journal of Science Culture and Sport (IntJSCS). 2015;3(2):64-72

5. da Mota GR, Thiengo CR, Gimenes SV, Bradley PS. The effects of ball possession status on physical and technical indicators during the 2014 FIFA World Cup Finals. J Sports Sci. 2016;34(6):493-500. Epub 2015/12/24 
6. Bertolaccini MdS, Orsatti FL, Barbosa Neto O, Mendes EL, Penaforte FRdO, Ide BN, et al. Soccer only once a week generates excessive cardiac responses. J Health Sci Inst. 2010;28(3):272-4.

7. Mota GR, Gomes LH, Castardeli E, Bertoncello D, Vicente EJD, Marocolo $\mathrm{M}$, et al. Treinamento proprioceptivo e de força resistente previnem lesões no futebol. J Health Sci Inst. 2010;28(2):191-3.

8. Møllerløkken NE, Lorås H, Pedersen AV. A SYSTEMATIC REVIEW AND META-ANALYSIS OF DROPOUT RATES IN YOUTH SOCCER. Percept Mot Skills. 2015;121(3):913-22. Epub 2015/11/23.

9. Wilbert-Lampen U, Leistner D, Greven S, Pohl T, Sper S, Volker C, et al. Cardiovascular events during World Cup soccer. The New England journal of medicine. 2008:358(5):475-83. Epub 2008/02/01.

10. Simim MA, Bradley PS, da Silva BV, Mendes EL, de Mello MT, Marocolo $\mathrm{M}$, et al. The quantification of game-induced muscle fatigue in amputee soccer players. J Sports Med Phys Fitness. 2017;57(6):766-72. Epub 2016/03/10.

11. Simim MAM, da Mota GR, Marocolo M, da Silva BVC, de Mello MT, Bradley PS. The Demands of Amputee Soccer Impair Muscular Endurance and Power Indices But Not Match Physical Performance. Adapt Phys Activ Q. 2018;35(1):76-92. Epub 2018/01/05.

12. Simim MAM, Silva BVC, Marocolo Júnior M, Mendes EL, Mello MTd, Mota GR. Anthropometric profile and physical performance characteristic of the Brazilian amputee football (soccer) team. Motriz: Revista de Educação Física. 2013;19:641-8.

13. Vicente-Vila $P$, Lago-Penas $C$. The goalkeeper influence on ball possession effectiveness in futsal. J Hum Kinet. 2016;51:217-24. Epub 2017/02/06.
14. Schuth G, Carr G, Barnes C, Carling C, Bradley PS. Positional interchanges influence the physical and technical match performance variables of elite soccer players. J Sports Sci. 2016;34(6):501-8. Epub 2015/12/24.

15. Anderson C, Sally D. Os números do jogo: porque tudo que você sabe sobre o futebol esta errado. $1^{\mathrm{a}}$ ed. São Paulo.: Paralela.; 2013. $201 \mathrm{p}$

16. Vendite LL, Moraes ACd, Vendite CC. Scout no futebol: uma análise estatística. Conexões. 2015;1(2):12. Epub 2015-09-21.

17. Mota GR, Magalhães CG, Azevedo PHSM, Ide BN, Lopes CR, Castardeli $E$, et al. Lactate threshold in taekwondo through specifics tests. Journal of Exercise Physiology online. 2011;14(3):60-6.

18. Cruz TMFd, Germano MD, Crisp AH, Sindorf MAG, Verlengia R, da Mota $\mathrm{GR}$, et al. Does Pilates Training Change Physical Fitness in Young Basketball Athletes? Journal of Exercise Physiology Online. 2014;17(1):19.

19. da Silva BV, Simim MA, Marocolo M, Franchini E, da Mota GR. Optimal load for the peak power and maximal strength of the upper body in Brazilian Jiu-Jitsu athletes. Journal of strength and conditioning research National Strength \& Conditioning Association. 2015;29(6):1616-21. Epub 2014/12/09.

20. Cohen J. Statistical power analysis for the behavioral sciences. $2^{\underline{a}}$ ed. Hillsdale: Lawarence Erlbaum Associates; 1998. 120 p.

21. Batterham AM, Hopkins WG. Making meaningful inferences about magnitudes. International Journal of Sports Physiology and Performance. 2006;1:50-7.

22. Bradley PS, Ade JD. Are Current Physical Match Performance Metrics in Elite Soccer Fit for Purpose or is the Adoption of an Integrated Approach Needed? Int J Sports Physiol Perform. 2018:1-23. Epub 2018/01/18. 